\title{
ON NORMAL RIEMANNIAN HOMOGENEOUS SPACES OF RANK 1
}

\author{
BY ISAAC CHAVEL ${ }^{1}$
}

Communicated by E. Calabi, January 5, 1967

In this note we shall prove (cf. definitions in [2]) the following

THEOREM. Let $G / H$ be a simply connected normal Riemannian homogeneous space of rank 1 such that every point $Q$ conjugate to $P_{0}=\pi(H)$ ( $\pi$ is the natural projection) is isotropically conjugate to $P_{0}$; then $G / H$ is homeomorphic to a Riemannian symmetric space of rank 1.

1. Preliminaries. M. Berger [1] has classified the simply connected normal Riemannian homogeneous spaces of rank 1, and with the exception of two, viz, $\mathrm{Sp}(2) / \mathrm{SU}(2)$ and $\mathrm{SU}(5) /(\mathrm{Sp}(2) \times \mathrm{T})$ all are homeomorphic to a Riemannian symmetric space of rank 1 . To prove the theorem it therefore suffices to exhibit in each of the spaces $\mathrm{Sp}(2) / \mathrm{SU}(2), \mathrm{SU}(5) /(\mathrm{Sp}(2) \times \mathrm{T})$ a conjugate point of $P_{0}=\pi(H)$ at which no isotropic Jacobi field vanishes. (A Jacobi field along a geodesic $\sigma(s)\left(\sigma(0)=P_{0}\right)$ is isotropic, if it is induded by a 1-parameter subgroup of $H$. A point at which at least one isotropic Jacobi field vanishes is said to be isotropically conjugate to $P_{0}$.) Furthermore, since the zeros (if they exist) of an isotropic Jacobi field occur only at in tegral multiples of a fixed real number (Lemma 1 of [2]), it suffices to exhibit in each case a Jacobi field along a geodesic emanating from $P_{0}$, vanishing for $s=\alpha$ and not for $s=2 \alpha$ ( $s=$ arc length along the geodesic), such that no Jacobi field with periodic zeros vanishes for $s=\alpha$. In [2] we exhibited the desired Jacobi field for the space $\mathrm{Sp}(2) / \mathrm{SU}(2)$; and we now do the same for the second example.

The equations we will solve will read as:

$$
d^{2} \eta_{\alpha} / d s^{2}+\left\langle Q_{\alpha},\left[\lambda, Q_{\beta}\right]\right\rangle\left(d \eta_{\beta} / d s\right)+\left\langle Q_{\alpha},\left[\left[\lambda, Q_{\beta}\right] \mathfrak{b}, \lambda\right]\right\rangle \eta_{\beta}=0,
$$

$\alpha, \beta$ (repeated indices summed) $=1, \cdots, n=\operatorname{dim} G / H$. In equation $(1), s$ denotes arc length along the geodesic, $\langle$,$\rangle the inner product on$ $\mathfrak{g}=\mathfrak{h}+\mathfrak{m}, \mathfrak{m}=\mathfrak{h}^{\perp}, Q_{\alpha}$ an orthonormal basis of $\mathfrak{m}$, and $\lambda \in \mathfrak{m}$ the initial unit velocity vector of the geodesic-as usual $\mathfrak{m}$ is identified with the tangent space of $P_{0}=\pi(H)[3]$. [ , ] is the Lie multiplication in $\mathfrak{g}$, and $[,]_{\mathfrak{b}}$ its projection onto $\mathfrak{h}$. We note that the matrices

$$
\begin{aligned}
T_{\alpha \beta}(\lambda) & =\left\langle Q_{\alpha},\left[\lambda, Q_{\beta}\right]\right\rangle, \\
K_{\alpha \beta}(\lambda) & =\left\langle Q_{\alpha},\left[\left[\lambda, Q_{\beta}\right]_{\mathfrak{b}}, \lambda\right]\right\rangle
\end{aligned}
$$

1 Partially supported by NSF Grant GP5760. 
are respectively skew-symmetric and symmetric [2].

Equation (1) is Jacobi's equation of geodesic deviation written in the canonical connection (of the second kind [3]) - which has the same geodesics as the metric connection-and with respect to an orthonormal frame $\left\{e_{\alpha}(s)\right\}$ along the geodesic such that: (i) $e_{\alpha}(0)$ $=Q_{\alpha}, \alpha=1, \cdots, n$, and (ii) for each $\alpha$, the field $e_{\alpha}(s)$ is parallel in the canonical connection [2]. The vanishing of the covariant derivatives of the torsion and curvature tensors of the canonical connection guarantees that the coefficients are constant. For the Riemannian symmetric case, where the metric and the canonical connections coincide, see [4].

We now turn to the space $\mathrm{SU}(5) /(\mathrm{Sp}(2) \times \mathrm{T})$.

2. Proof of the theorem. We first calculate the coefficients of Jacobi's equation with respect to the Lie algebra couple $\left(\boldsymbol{A}_{4}, C_{2} \oplus R\right)$. Let $E_{j k}$ be the matrix with a 1 in the $j$ th row and $k$ th column and 0 elsewhere: $E_{j k}=\left(\delta_{j r} \delta_{k s}\right)$. Then, setting

$i^{2}=-1, \quad A_{j k}=\left(E_{j j}-E_{k k}\right), \quad B_{j k}=E_{j k}-E_{k j}, \quad C_{j k}=i\left(E_{j k}+E_{k j}\right)$

we have

$$
\begin{aligned}
& {\left[A_{r j}, A_{k l}\right]=-\delta_{r k} B_{r k}+\delta_{r l} B_{r l}+\delta_{j k} B_{j k}-\delta_{j l} B_{j l},} \\
& {\left[A_{r j}, B_{k l}\right]=\delta_{r k} C_{r l}-\delta_{r l} C_{r k}-\delta_{j k} C_{j l}+\delta_{j l} C_{j k},} \\
& {\left[A_{r j}, C_{k l}\right]=-\delta_{r k} B_{r l}-\delta_{r l} B_{r k}+\delta_{j k} B_{j l}+\delta_{j l} B_{j k},} \\
& {\left[B_{r j}, B_{k l}\right]=\delta_{j k} B_{r l}-\delta_{j l} B_{r k}-\delta_{r k} B_{j l}+\delta_{r l} B_{j k}} \\
& {\left[B_{r j}, B_{k l}\right]=\delta_{j l} C_{r k}+\delta_{j k} C_{r l}-\delta_{r l} C_{j k}-\delta_{r l} C_{j l},} \\
& {\left[C_{r j}, C_{k l}\right]=-\delta_{j k} B_{r l}-\delta_{j l} B_{r k}-\delta_{r k} B_{j l}-\delta_{r l} B_{j k} .}
\end{aligned}
$$

Furthermore, setting $\langle X, Y\rangle=-\frac{1}{2}$ trace $(X, Y), X, Y \in A_{4}$, we obtain an orthogonal decomposition for the couple $\left(\boldsymbol{A}_{4}, \boldsymbol{C}_{\mathbf{2}} \oplus R\right)$ :

$$
\begin{aligned}
& S_{1}=A_{12} ; \quad S_{2}=B_{12} ; \quad S_{3}=B_{13} ; \quad S_{4}=\left(B_{13}-B_{24}\right) / 2^{1 / 2} ; \\
& S_{5}=\left(C_{13}+C_{24}\right) / 2^{1 / 2} ; \\
& S_{6}=\left(B_{14}+B_{23}\right) / 2^{1 / 2} ; \quad S_{7}=\left(C_{14}-C_{23}\right) / 2^{1 / 2} ; \quad S_{8}=A_{34} ; \quad S_{9}=B_{34} ; \\
& S_{10}=C_{34} ; \quad S_{11}=2\left(A_{15}-A_{12} / 2\right) / 3^{1 / 2} ; \\
& Q_{1}=3^{1 / 2} A_{13} / 2^{1 / 2}-A_{12} / 6^{1 / 2}-A_{15} / 6^{1 / 2} ; \quad Q_{2}=\left(B_{13}+B_{24}\right) / 2^{1 / 2} ; \\
& Q_{3}=\left(C_{13}-C_{24}\right) / 2^{1 / 2} ; \quad Q_{4}=\left(B_{14}-B_{23}\right) / 2^{1 / 2} ; Q_{5}=\left(C_{14}+C_{23}\right) / 2^{1 / 2} ; \\
& Q_{6}=B_{15} ; \quad Q_{7}=C_{15} ; \quad Q_{8}=B_{25} ; \quad Q_{9}=C_{25} ; \quad Q_{10}=B_{35} ; Q_{11}=C_{35}, \\
& Q_{12}=B_{45} ; \quad Q_{13}=C_{45}
\end{aligned}
$$


where $S_{1}, \cdots, S_{11}$, span $C_{2} \oplus R$, and $Q_{1}, \cdots, Q_{13}$ span $\mathfrak{m}=\left(C_{2} \oplus R\right)^{\perp}$.

We let $\sigma$ be the geodesic emanating from $P_{0}$, given by $\sigma(s)$ $=\pi\left(\exp \left(s Q_{6}\right)\right)$. Then for the torsion terms $T_{\alpha \beta}\left(Q_{6}\right)$ we have: $T_{17}$ $=-T_{71}=1 / 6^{1 / 2} ; \quad-T_{2,10}=T_{10,2}=T_{3,11}=-T_{11,8}=-T_{4,12}=T_{12,4}=T_{5,13}$ $=-T_{18,5}=1 / 2^{1 / 2}$; all the rest are zero. For the curvature terms $K_{\alpha \beta}\left(Q_{6}\right)$ we have $K_{77}=4 ; K_{88}=K_{99}=K_{10,10}=1 ; K_{11,11}=K_{12,12}=K_{18,13}$ $=1 / 2$; all the rest are zero.

The associated characteristic polynomial of (1) along $\sigma(s)$ $=\pi\left(\exp \left(s Q_{6}\right)\right)$ is then calculated to be

$$
\begin{aligned}
0 & =\operatorname{det}\left(x^{2} I+x T+K\right) \\
& =x^{12}\left(x^{2}+1\right)^{5}\left(x^{2}+3 / 2\right)\left(x^{2}+25 / 6\right) .
\end{aligned}
$$

A basis of solutions, $\eta(s)$, of (1) satisfying $\eta(0)=0$ is given by:

$$
\begin{aligned}
& \eta_{1}(s)=\alpha_{1}\left(1-\cos \left(5 s / 6^{1 / 2}\right)\right)+\alpha_{2}\left(20\left(6^{1 / 2}\right) s+\sin \left(5 s / 6^{1 / 2}\right)\right), \\
& \eta_{2}(s)=\alpha_{3}\left(1-\cos \left(3^{1 / 2} s / 2^{1 / 2}\right)\right)+\alpha_{4}\left(6^{1 / 2} s+\sin \left(3^{1 / 2} s / 2^{1 / 2}\right)\right), \\
& \eta_{3}(s)=\alpha_{5}(1-\cos s)+\alpha_{6}(s+\sin s), \\
& \eta_{4}(s)=\alpha_{7}(1-\cos s)+\alpha_{8}(s+\sin s), \\
& \eta_{5}(s)=\alpha_{9}(1-\cos s)+\alpha_{10}(s+\sin s), \\
& \eta_{6}(s)=\alpha_{11} s, \\
& \eta_{7}(s)=-5 \alpha_{1} \sin \left(5 s / 6^{1 / 2}\right)+5 \alpha_{2}\left(1-\cos \left(5 s / 6^{1 / 2}\right)\right), \\
& \eta_{8}(s)=\alpha_{12} \sin s \\
& \eta_{9}(s)=\alpha_{13} \sin s \\
& \eta_{10}(s)=3^{1 / 2} \alpha_{3} \sin \left(3^{1 / 2} s / 2^{1 / 2}\right)-3^{1 / 2} \alpha_{4}\left(1-\cos \left(3^{1 / 2} s / 2^{1 / 2}\right)\right), \\
& \eta_{11}(s)=-2^{1 / 2} \alpha_{5} \sin s+2^{1 / 2} \alpha_{6}(1-\cos s), \\
& \eta_{12}(s)=2^{1 / 2} \alpha_{7} \sin s-2^{1 / 2} \alpha_{8}(1-\cos s), \\
& \eta_{13}(s)=-2^{1 / 2} \alpha_{9} \sin s+2^{1 / 2} \alpha_{10}(1-\cos s) .
\end{aligned}
$$

Now, as we shall show (cf. Remark), the subspace of the isotropic Jacobi fields along $\pi\left(\exp \left(s, Q_{6}\right)\right)$ are spanned by the solutions $\alpha_{k}=\delta_{j k}$, $j=1,3,7,12$. Furthermore there exist $\alpha_{5}, \alpha_{6}$ such that $\alpha_{5} \alpha_{6} \neq 0$ and the solution $\eta\left(s ; 0, \cdots, 0, \alpha_{5}, \alpha_{6}, 0, \cdots, 0\right)$ of (1) has zeros. The values, $\alpha$, of $s$ for which $\eta(s)=0$ are the zeros $\alpha$ of the determinant

$$
\left|\begin{array}{cc}
1-\cos s & s+\sin s \\
-\sin s & 1-\cos s
\end{array}\right|
$$

$\equiv f(s)=2(1-\cos s)+s \cdot \sin s$ such that $\alpha \neq 2 \pi k$ where $k$ is any integer. Note that $f(0)=0, f(s)>0,0<s<\pi, f(\pi)=4, f(4 \pi / 3)<0$, which im- 
plies there exists $\alpha, \pi<\alpha<4 \pi / 3$ such that $f(\alpha)=0$. The zeros of the isotropic Jacobi fields are at $2 \pi(6)^{1 / 2} / 5, \pi, 2 \pi(2)^{1 / 2} / 3^{1 / 2}, 4 \pi(6)^{1 / 2} / 5$, $2 \pi$, and higher value of $s$. $6^{1 / 2}<5 / 2$ implies $(2 \pi / 5) 6^{1 / 2}<\pi$; and $(4 \pi / 5) 6^{1 / 2}>8 \pi / 5>4 \pi / 3$; and $2(2)^{1 / 2} \pi / 3^{1 / 2}>4 \pi / 3$, which implies that no isotropic Jacobi field vanishes for $s=\alpha$-and the theorem is proven.

Remark. Let $\sigma(s)=\pi(\exp (s B))$ be a geodesic emanating from $P_{0}$ in a reductive homogeneous space $G / H$, with Lie algebra decomposition $\mathfrak{g}=\mathfrak{h}+\mathfrak{m}, B \in \mathfrak{m}$, and let $v(s, \epsilon)$ be the isotropic variation of $\sigma$ given by $v(s, \epsilon) \equiv \tau(\exp (\epsilon A)) \cdot \pi(\exp (s B)), A \in \mathfrak{h}$. Then

$$
d(d \tau(\exp \epsilon A) \cdot B) / d \epsilon=[A, B]
$$

where the above derivative is taken in the vector space $m$; and

$$
d(d \tau(\exp \epsilon A) \cdot B) / d \epsilon=\delta \eta /\left.d s\right|_{s=0}=D \eta /\left.d s\right|_{s=0},
$$

where $\eta$ is the induced isotropic Jacobi field of $v(s, \epsilon), \delta / d s$ is $\omega$-variant differentiation along $\sigma(s)$ with respect to the Cartan connection and $D / d s$ is covariant differentiation along $\sigma(s)$ with respect to the canonical connection.

Therefore, in the above example (\$2), since

$$
\begin{aligned}
{\left[3^{1 / 2} S_{1}-S_{11}, Q_{6}\right] } & =\left[S_{2}-S_{3}, Q_{6}\right]=\left[S_{4}-S_{a}, Q_{6}\right]=\left[S_{6}-S_{7}, Q_{6}\right] \\
& =\left[S_{8}, Q_{6}\right]=\left[S_{9}, Q_{6}\right]=\left[S_{10}, Q_{6}\right]=0,
\end{aligned}
$$

the corresponding isotropic variations of $\pi\left(\exp \left(s Q_{6}\right)\right)$ are all trivial, i.e., they leave the geodesic $\pi\left(\exp \left(s Q_{6}\right)\right)$ fixed. The nontrivial isotropic variations are generated by the linear span of $S_{1},-\left(S_{2}+S_{3}\right) / 2$, $-\left(S_{4}+S_{5}\right) / 2^{1 / 2},-\left(S_{6}+S_{7}\right) / 2^{1 / 2}$ and $\delta \eta / d s_{s-0}$ is given respectively by

$$
\begin{aligned}
{\left[S_{1}, Q_{6}\right] } & =Q_{7}, \\
{\left[-\left(S_{2}+S_{8}\right) / 2^{1 / 2}, Q_{6}\right] } & =Q_{8}, \\
{\left[-\left(S_{4}+S_{5}\right) / 2^{1 / 2}, Q_{6}\right] } & =Q_{10}, \\
{\left[-\left(S_{6}+S_{7}\right) / 2^{1 / 2}, Q_{6}\right] } & =Q_{12}
\end{aligned}
$$

with corresponding constants of integration $\alpha_{k}=c_{j} \delta_{j k}, j=1,12,3,7$ respectively, where $c_{j}$ are constants.

\section{REFERENCES}

1. M. Berger, Les variêtés Riemannienes homogènes normales simplement connexes a courbure strictement positive, Ann. Scuola Norm. Sup. Pisa 15 (1961), 174-246.

2. I. Chavel, Isotropic Jacobi fields, and Jacobi's equations on Riemannian homogeneous spaces, Comment Math. Helv. (to appear). 
3. K. Nomizu, Invariant affine connections on homogeneous spaces, Amer. J. Math. 76 (1954), 33-65.

4. H. E. Rauch, Geodesics and Jacobi's equations on Riemannian homogeneous spaces, pp. 115-127. Proc. U. S.-Japan Seminar in differential geometry, Kyoto U., 1965.

UNIVERSITY OF MINNESOTA 